\title{
State and trait anxiety in islander cardiac patients: associated factors and the impact of perceived social support
}

Fotini Legaki, Fotoula Babatsikou, Charilaos Koutis, Maria Polikandrioti

Laboratory and Clinical Nursing Cardiology, Attikon Hospital, Greece

Submitted: 20 March 2020

Accepted: 27 April 2020

Arch Med Sci Atheroscler Dis 2020; 5: e85-e97

DOI: https://doi.org/10.5114/amsad.2020.95877

Copyright @ 2020 Termedia \& Banach

\begin{abstract}
Introduction: Cardiac patients frequently experience elevated levels of anxiety, which may influence clinical outcomes. Perceived social support is a precious resource with which to minimise anxiety. The aim of the study was to explore levels of perceived social support, levels of anxiety (state and trait), and the associated factors as well as the impact of patents' characteristics and social support on anxiety.

Material and methods: The study sample consisted of 82 island inhabitant patients with cardiac problems (49 men and 33 women). Data collected by the completion of the State-trait anxiety inventory (STAI) and the Multidimensional scale of perceived social support questionnaire (MSPSS). The statistical significance level was set at $p<0.05$.

Results: Regarding state and trait anxiety, half of the patients scored below 37 and 38, respectively, (medians) while $25 \%$ of participants scored more than 50 and 48, respectively. These values indicate moderate levels of anxiety. Regarding social support, at least $50 \%$ of patients scored over 27 , 27 , and 20 (median) on support from significant ones, family, and friends, respectively. In addition, $25 \%$ of patients scored above 28,28 , and 26 , respectively. These values indicate high levels of social support. The association between anxiety and social support was found to be linearly negative, meaning that the more support a patient felt, the less anxiety they also experienced.
\end{abstract}

Conclusions: Enhancing perceived social support may be a key-element in alleviating anxiety among cardiac patients.

Key words: perceived social support, state, trait anxiety, cardiac disease.

\section{Introduction}

During recent decades the life expectancy of cardiac patients has been prolonged due to considerable advances in the field of treatment, including progress in diagnostic tools and improvements in coronary intervention. Meanwhile, the role of demographic characteristics in the manifestation and outcome of cardiac diseases are well documented, and these parameters are evaluated in daily clinical practice [1, 2].

Furthermore, cardiac diseases often occur in parallel with psychological distress [3]. A high prevalence of anxiety is observed in many types of cardiac disease, either chronic or acute. For instance, anxiety is prevalent in $20-30 \%$ of patients after an acute coronary syndrome, which persists in half of cases up to one year post-event $[1,2]$.

\author{
Corresponding author: \\ Fotini Legaki \\ Laboratory and Clinical \\ Nursing Cardiology \\ Attikon Hospital \\ E-mail: \\ fwtoulanaxos@yahoo.gr
}


Association between anxiety and cardiac disease is two faceted. Anxiety is associated with increased risk of developing cardiac disease or the deterioration of an already established one [1].

On the other hand, patients with anxiety may experience cardiovascular system symptoms, or a cardiac disease may underlie in patients with psychiatric symptoms [4-6].

Anxiety incorporates two complementary concepts: the psychophysiological state (state anxiety) and the personality trait (trait anxiety). State anxiety evaluates the current condition of anxiety or transient reaction directly related to adverse situations in a specific moment. In contrast, trait anxiety evaluates stable aspects of "anxiety proneness", thus describing an individual's tendency [7].

Nowadays, there is increasing effort to enable patients experiencing psychological distress to assume responsibility for their own health management. Therefore, it is essential in clinical settings to differentiate between temporary and long-standing conditions of anxiety.

Perceived social support has beneficial clinical implications in several chronic illness, such as haemodialyis [8], diabetic ulcer [9], and cardiac disease $[10,11]$. Low social support along with depression, which often co-exists with anxiety, are significant indicators for poor prognosis in cardiac patients [11].

Undoubtedly, living on an island is associated with several barriers. The characteristics of sea islanders, such as low rates of smoking, increased daily physical activity, and healthy eating habits, are among those that contribute to longevity; however, a wide range of factors may hamper their heath, such as health care deficiencies or failure of early access to optimal healthcare, reluctance to report increased stress for fear of stigmatisation, and several others [12].

A broader understanding of factors influencing anxiety among cardiac patients living on an island as well as the impact of social support on anxiety may serve to alert clinicians in anxiety-related areas that would otherwise be overlooked.

To the best of our knowledge, data exploring anxiety among cardiac patients living on an island and the associated factors are limited.

Thus, the aim of this cross-sectional study was to explore levels of perceived social support and levels of anxiety (state and trait) and the associated factors, as well as the impact of patents' characteristics and social support on anxiety.

\section{Material and methods}

\section{Study population}

The sample of the study consisted of 82 patients with cardiac problems, living on the island of Naxos (49 men and 33 women). It was a convenience sample.

Criteria for inclusion in the study were as follows: a) an established cardiac disease and not a first diagnosis, b) the ability to write and read the Greek language fluently, and c) permanent residency on the island.

In greater detail, cardiac disease had already been established and participants had received treatment for their cardiac disease in urban areas, mainly in Athens. The patients were included in the present study because they lived permanently on the island and they regularly visited the outpatient department of the public hospital for monitoring and follow-up.

The exclusion criteria were patients with the following: a) a history of mental illness and b) other chronic disease.

Data collection was performed by interview method using a questionnaire, which included the following: a) socio-demographic, clinical, and other patients' self-reported characteristics; b) the State-Trait Anxiety Inventory (STAI); and c) the Multidimensional Scale of Perceived Social Support questionnaire (MSPSS).

Completion of the questionnaires lasted approximately 15 min and took place after patients had finished their regular monitoring and follow-up.

In the present study there was no intervention or control group because this research merely recorded whether patients experienced anxiety and its association with social support. Literature on this association was limited and did not enable extensive comparative research.

\section{Ethical considerations}

Patients who met the entry criteria were informed by the researcher for the purposes of the study and participated only after they had given their written consent. All of the patients participated in the study on a voluntary basis and had their anonymity preserved. All participants were informed of their rights to refuse or to discontinue their participation, according to the ethical standards of the Helsinki Declaration of 1983. The study was approved by the Medical Research Ethics Committee of the hospital.

\section{Demographic characteristics}

The study collected the following socio-demographic characteristics of the participants: gender, age, marital and educational status, and job.

\section{Clinical characteristics}

In addition, data on the underlying disease of the participants were collected. More specifically, the following items were recorded: the type of car- 
diac disease they were suffering from, the number of years since the onset of their cardiac problem, whether they were suffering from another disease, whether they reported insomnia.

\section{Other self-reported characteristics}

Patients were also asked about the following:

- Their level of information about cardiac disease.

- The need for written information about their cardiac disease.

- Difficulties in their everyday activities.

- Limitations in social contact due to cardiac disease.

- Their relationship with nursing, medical staff, and other patients.

- Whether they experienced dependency on health professionals.

- Whether they experienced support from their family after diagnosis.

- Whether they were satisfied with their life.

- Whether they experienced insecurity due to their life on an island.

\section{State and trait anxiety assessment}

The State-Trait Anxiety Inventory (STAI) scale was used to assess anxiety among cardiac islanders. State-trait anxiety scales (temporary and permanent anxiety) consist of 20 questions (respectively) that evaluate how respondents feel at the time of completing the questionnaire. Respondents were able to answer each question on a Likert-type scale (scores of 1-4). The scores attributed to the questions are summed, leading to a final score of temporary and permanent anxiety, respectively. Higher score values indicate higher levels of anxiety [13].

\section{Perceived social support assessment}

The Multidimensional Scale of Perceived Social Support questionnaire (MSPSS) was used to evaluate perceived social support. This scale had been translated and culturally adapted to Greek standards. It assesses three dimensions of social support: support from significant others, family, and friends. The questions of each dimension expressing "support" are rated at a seven-point Likert scale from 1 to 7 . In order to calculate the final score of each dimension of perceived social support, we add the scores of questions corresponding to each dimension and divide by the number of questions included in each dimension. These scores reflect the degree of support felt by the patients. Higher scores indicate higher support [14].

\section{Statistical analysis}

Categorical data are presented with absolute and relative (\%) frequencies, whereas continuous data are presented with mean, standard deviation, median, and interquartile range. The normality of the data was verified by Kolmogorov-Smirnov test and graphically with histograms and Q-Q plots. The Mann-Whitney and Kruskal-Wallis tests were used to evaluate the association between anxiety scores, social support, and the patient's characteristics. Levels of anxiety and perceived social support were estimated by both mean and standard deviation (SD) and median and interquartile range (IQR).

Multiple linear regression was applied to assess the effect of the characteristics on the state and trait anxiety as well as the social support of patients. Results are presented with $\beta$ coefficients and $95 \%$ confidence intervals. The observed significance level of $5 \%$ was considered statistically significant. All statistical analyses were performed with SPSS version 25 (SPSS Inc., Chicago, IL, USA).

\section{Results}

\section{Sample description}

Table I presents the demographic characteristics of the sample. In particular, men accounted for $59.8 \%$ of the sample, $32.9 \%$ were over 70 years of age, $67.1 \%$ were married, $37.8 \%$ had primary education, and $42.7 \%$ were retired.

Table II presents data related to patients' clinical characteristics. The majority of patients had myocardial infarction (34.1\%) and atrial fibrillation $(24.4 \%)$. Furthermore, in $28 \%$ of participants, the onset of cardiac disease was between 2 and 5 years, in $88.9 \%$ it co-existed some other disease, and $34.1 \%$ reported insomnia.

Table III presents other self-reported characteristics. In greater detail, 32.9\% declared they were "very" well informed about their cardiac disease and $63.4 \%$ reported the need for written information about their cardiac disease. Difficulties in everyday activities accounted for $37.8 \%$ of the sample, while half of the patients (51.2\%) did not limit their social contacts "at all" due to their cardiac disease. Moreover, $69.5 \%, 73.2 \%$, and $32.9 \%$ of patients had "very good" relations with nursing, medical staff, and other patients, respectively.

Additionally, $56.1 \%$ believed that their life depended on health professionals, $69.5 \%$ believed their family supported them "very much", and $24.4 \%$ declared they were "very" satisfied with their life. Finally, the majority of the patients $(65.9 \%)$ stated that they experienced insecurity because they were living on an island.

\section{Measurement of state and trait anxiety}

The results in Table IV refer to the state-trait anxiety of patients. We can observe that half of patients scored below 37 and 38 in state and trait anxiety, respectively (median), while $25 \%$ of the 
Table I. Demographic characteristics $(N=82)$

\begin{tabular}{|c|c|}
\hline Parameter & $N(\%)$ \\
\hline \multicolumn{2}{|l|}{ Gender: } \\
\hline Male & $49(59.8)$ \\
\hline Female & $33(40.2)$ \\
\hline \multicolumn{2}{|l|}{ Age: } \\
\hline$<30$ & $2(2.4)$ \\
\hline $30-40$ & $8(9.8)$ \\
\hline $41-50$ & $11(13.4)$ \\
\hline $51-60$ & $14(17.1)$ \\
\hline $61-70$ & $20(24.4)$ \\
\hline$>70$ & $27(32.9)$ \\
\hline \multicolumn{2}{|l|}{ Family status: } \\
\hline Married & $55(67.1)$ \\
\hline Single & $8(9.8)$ \\
\hline Divorced & $6(7.3)$ \\
\hline Widowed & $9(11.0)$ \\
\hline Living together & $4(4.9)$ \\
\hline \multicolumn{2}{|l|}{ Education: } \\
\hline Primary school & $31(37.8)$ \\
\hline High school & $33(40.2)$ \\
\hline University & $14(17.1)$ \\
\hline$M S c-P h D$ & $4(4.9)$ \\
\hline \multicolumn{2}{|l|}{ Job: } \\
\hline Unemployed & $3(3.7)$ \\
\hline Civil servant & $9(11.0)$ \\
\hline Private employee & $7(8.5)$ \\
\hline Freelancer & $15(18.3)$ \\
\hline Household & $9(11.0)$ \\
\hline Pensioner & $35(42.7)$ \\
\hline Other & $4(4.9)$ \\
\hline
\end{tabular}

patients scored more than 50 and 48 , respectively. These values, in relation to the possible range of anxiety scores, indicate moderate levels of state and trait anxiety in patients. Also, the results are presented in mean and standard deviation (SD).

\section{Measurement of perceived social support}

The results in Table $V$ refer to perceived social support experienced by patients with cardiac problems. We note that at least $50 \%$ of patients scored over 27, 27, and 20 (median) regarding
Table II. Clinical characteristics $(N=82)$

\begin{tabular}{|c|c|}
\hline Parameter & $N(\%)$ \\
\hline \multicolumn{2}{|l|}{ Cardiac disease: } \\
\hline Heart failure & $15(18.3)$ \\
\hline Myocardial infraction & $28(34.1)$ \\
\hline Atrial fibrillation & $20(24.4)$ \\
\hline Unstable angina & $12(14.6)$ \\
\hline Pacemaker & $7(8.5)$ \\
\hline \multicolumn{2}{|c|}{$\begin{array}{l}\text { Number of years since the onset } \\
\text { of cardiac disease: }\end{array}$} \\
\hline$<1$ & $22(26.8)$ \\
\hline $2-5$ & $23(28.0)$ \\
\hline $6-10$ & $20(24.4)$ \\
\hline $11-15$ & $4(4.9)$ \\
\hline$>15$ & $13(15.9)$ \\
\hline \multicolumn{2}{|l|}{ Other disease: } \\
\hline Yes & $72(88.9)$ \\
\hline No & $9(11.1)$ \\
\hline \multicolumn{2}{|l|}{ Insomnia: } \\
\hline Yes & $28(34.1)$ \\
\hline No & $54(65.9)$ \\
\hline
\end{tabular}

support from significant ones, family, and friends, respectively. In addition, $25 \%$ of patients scored above 28,28 , and 26 , respectively. These values, in relation to the possible score range (4-28), indicate high levels of perceived social support for patients. Patients experienced more social support from significant ones and family and less from friends. Also, the results are presented as mean and standard deviation (SD).

\section{Association between patients' characteristics and state and trait anxiety}

Table VI presents the association between patient's characteristics and anxiety (state and trait).

Both measurements (state and trait anxiety) were found to be statistically significantly associated with the existence of other diseases ( $p=$ 0.037 and $p=0.026$, respectively), whether they had insomnia $(p=0.037$ and $p=0.016$, respectively), whether they experienced difficulties in everyday activities ( $p=0.032$ and $p=0.027$, respectively), whether they had limited their social contact ( $p=0.004$ and $p=0.025$, respectively), and how satisfied they were with their lives ( $p$ $=0.001$ and $p=0.001$, respectively). More specifically, patients with another disease had more 
Table III. Other self-reported characteristics $(N=82)$

\begin{tabular}{|c|c|}
\hline Parameter & $N(\%)$ \\
\hline \multicolumn{2}{|c|}{ Level of information about cardiac disease: } \\
\hline A lot & $27(32.9)$ \\
\hline Enough & $46(56.1)$ \\
\hline A little & $9(11.0)$ \\
\hline None at all & $0(0.0)$ \\
\hline \multicolumn{2}{|c|}{$\begin{array}{l}\text { Do you need written information about your cardiac } \\
\text { disease? }\end{array}$} \\
\hline Very much & $52(63.4)$ \\
\hline Enough & $22(26.8)$ \\
\hline A little & $8(9.8)$ \\
\hline Not at all & $0(0.0)$ \\
\hline \multicolumn{2}{|c|}{ Difficulties in everyday activities: } \\
\hline Yes & $31(37.8)$ \\
\hline No & $51(62.2)$ \\
\hline \multicolumn{2}{|c|}{$\begin{array}{l}\text { Have you limited your social contact due to your cardiac } \\
\text { disease? }\end{array}$} \\
\hline Very much & $5(6.1)$ \\
\hline Enough & $11(13.4)$ \\
\hline A little & $24(29.3)$ \\
\hline Not at all & $42(51.2)$ \\
\hline \multicolumn{2}{|c|}{ Relations with nursing staff: } \\
\hline Very good & $57(69.5)$ \\
\hline Good & $22(26.8)$ \\
\hline Moderate & $2(2.4)$ \\
\hline $\mathrm{Bad}$ & $1(1.2)$ \\
\hline \multicolumn{2}{|c|}{ Relations with medical staff: } \\
\hline Very good & $60(73.2)$ \\
\hline Good & $19(23.2)$ \\
\hline
\end{tabular}

\begin{tabular}{|lc|}
\hline Parameter & $\boldsymbol{N}(\%)$ \\
\hline Moderate & $3(3.7)$ \\
\hline Bad & $0(0.0)$ \\
\hline Relations with other patients: \\
\hline Very good & $27(32.9)$ \\
\hline Good & $40(48.8)$ \\
\hline Moderate & $15(18.3)$ \\
\hline Bad & $0(0.0)$ \\
\hline
\end{tabular}

You believe that your life depends on healthcare professionals?

\begin{tabular}{lc}
\hline Very much & $46(56.1)$ \\
\hline Enough & $30(36.6)$ \\
\hline Little & $6(7.3)$ \\
\hline Not at all & $0(0.0)$
\end{tabular}

Do you believe your family supports you after the diagnosis?

\begin{tabular}{lc}
\hline Very much & $57(69.5)$ \\
\hline Enough & $20(24.4)$ \\
\hline A little & $2(2.4)$ \\
\hline Not at all & $3(3.7)$ \\
\hline How satisfied you are with your life? & \\
\hline Very much & $20(24.4)$ \\
\hline Enough & $47(57.3)$ \\
\hline A little & $10(12.2)$ \\
\hline Not at all & $5(6.1)$
\end{tabular}

Do you experience insecurity because you live on an island?

\begin{tabular}{ll}
\hline Yes & $54(65.9)$ \\
\hline No & $28(34.1)$ \\
\hline
\end{tabular}

Table IV. Measurement of patients' state and trait anxiety

\begin{tabular}{|llc|}
\hline Paramater & Mean (SD) & Median (IQR) \\
\hline State anxiety (range: 20-80) & $39.4(13.8)$ & $37(29-50)$ \\
\hline Trait anxiety (range: $20-80)$ & $39.5(11.0)$ & $38(31-48)$ \\
\hline
\end{tabular}

$S D$ - standard deviation, $I Q R$ - interquartile range.

Table V. Levels of perceived social support

\begin{tabular}{|lcc|}
\hline Support from & Mean (SD) & Median (IQR) \\
\hline Significant ones (range: 4-28) & $25.4(3.2)$ & $27(23-28)$ \\
\hline Family (range: 4-28) & $25.4(4.1)$ & $27(24-28)$ \\
\hline Friends (range: 4-28) & $20.9(5.6)$ & $20(16-26)$ \\
\hline
\end{tabular}

$S D$ - standard deviation, IQR - interquartile range.

state and trait anxiety (median 38 and 39.5, respectively) than patients without other disease (median 31 and 32, respectively). Patients who had insomnia (median 46 and 44.5, respectively) had more state and trait anxiety than those who did not have insomnia (median 34.5 and 35, respectively). Accordingly, patients who faced difficulties in everyday activities had more state and trait anxiety (median 44 and 43, respectively) than those who did not (median 35 and 35, re- 
Fotini Legaki, Fotoula Babatsikou, Charilaos Koutis, Maria Polikandrioti

Table VI. Association between patients' characteristics and state and trait anxiety

\begin{tabular}{|c|c|c|c|c|}
\hline Parameter & $\begin{array}{l}\text { State anxiety } \\
\text { Median (IQR) }\end{array}$ & $P$-value & $\begin{array}{l}\text { Trait anxiety } \\
\text { Median (IQR) }\end{array}$ & $P$-value \\
\hline Gender: & & 0.060 & & 0.055 \\
\hline Male & $33(26-46)$ & & $34(29-41)$ & \\
\hline Female & $41(35-52)$ & & $43(35-51)$ & \\
\hline Age: & & 0.309 & & 0.282 \\
\hline$<50$ & $37(31-42)$ & & $34(29-44)$ & \\
\hline $51-60$ & $41.5(35-51)$ & & $40(33-51)$ & \\
\hline $61-70$ & $31(23-44)$ & & $37.5(30.5-42.5)$ & \\
\hline$>70$ & $38(29-53)$ & & $43(34-51)$ & \\
\hline Family status: & & 0.936 & & 0.163 \\
\hline Married/living together & $37(29-48)$ & & $35(29-49)$ & \\
\hline Single/widowed/divorced & $38(29-52)$ & & $42(35-46)$ & \\
\hline Education: & & 0.303 & & 0.003 \\
\hline Primary school & $35(29-50)$ & & $43(35-51)$ & \\
\hline High school & $39(31-52)$ & & $36(31-44)$ & \\
\hline University & $34.5(27-40)$ & & $32.5(28-37)$ & \\
\hline Job: & & 0.800 & & 0.067 \\
\hline Unemployed/household & $43.5(30-51)$ & & $45(40.5-50.5)$ & \\
\hline Employee & $37(31-43)$ & & $34(29-44)$ & \\
\hline Pensioner & $34(25-52)$ & & $38(32-48)$ & \\
\hline Cardiac disease: & & 0.438 & & 0.898 \\
\hline Heart failure & $35(29-38)$ & & $38(34-41)$ & \\
\hline Myocardial infraction & $36.5(26-47)$ & & $35.5(29.5-51)$ & \\
\hline Atrial fibrillation & $37(30-45)$ & & $41(30-45)$ & \\
\hline Unstable angina & $42.5(30.5-53.5)$ & & $38(32.5-52.5)$ & \\
\hline Pacemaker & $55(23-64)$ & & $44(26-48)$ & \\
\hline $\begin{array}{l}\text { Number of years since the onset of cardiac } \\
\text { disease: }\end{array}$ & & 0.962 & & 0.347 \\
\hline$<1$ & $37(29-46)$ & & $33.5(29-44)$ & \\
\hline $2-5$ & $39(30-52)$ & & $41(31-45)$ & \\
\hline $6-15$ & $36.5(26.5-51)$ & & $39.5(33.5-47)$ & \\
\hline$>15$ & $35(31-48)$ & & $40(35-52)$ & \\
\hline Other disease: & & 0.037 & & 0.026 \\
\hline Yes & $38(30-51.5)$ & & $39.5(32-49)$ & \\
\hline No & $31(26-36)$ & & $32(28-35)$ & \\
\hline Insomnia: & & 0.037 & & 0.016 \\
\hline Yes & $46(33.5-54)$ & & $44.5(34-51.5)$ & \\
\hline No & $34.5(29-42)$ & & $35(29-43)$ & \\
\hline Level of information about cardiac disease: & & 0.730 & & 0.348 \\
\hline A lot & $36(27-46)$ & & $35(28-44)$ & \\
\hline Enough & $38(30-51)$ & & $40(32-48)$ & \\
\hline Little & $34(23-51)$ & & $38(35-51)$ & \\
\hline Need of receiving written information: & & 0.244 & & 0.529 \\
\hline Very much & $38(29.5-50.5)$ & & $40(32-47)$ & \\
\hline Enough & $35.5(29-52)$ & & $34(29-45)$ & \\
\hline Little & $30.5(29-33)$ & & $34.5(31.5-51)$ & \\
\hline
\end{tabular}


Table VI. Cont.

\begin{tabular}{|c|c|c|c|c|}
\hline Parameter & $\begin{array}{l}\text { State anxiety } \\
\text { Median (IQR) }\end{array}$ & $P$-value & $\begin{array}{l}\text { Trait anxiety } \\
\text { Median (IQR) }\end{array}$ & $P$-value \\
\hline Difficulties in everyday activities: & & 0.032 & & 0.027 \\
\hline Yes & $44(34-55)$ & & $43(34-51)$ & \\
\hline No & $35(29-43)$ & & $35(29-43)$ & \\
\hline Limited social contact: & & 0.004 & & 0.025 \\
\hline Very/enough & $47(32-57.5)$ & & $43(34-51)$ & \\
\hline Little & $44.5(34-52.5)$ & & $43.5(32.5-52.5)$ & \\
\hline Not at all & $33(23-39)$ & & $35(29-41)$ & \\
\hline Relations with nursing staff: & & 0.161 & & 0.574 \\
\hline Very good & $36(28-43)$ & & $38(32-45)$ & \\
\hline Good & $43.5(31.5-52.5)$ & & $36(28.5-50)$ & \\
\hline Relations with medical staff: & & 0.872 & & 0.114 \\
\hline Very good & $37(28.5-49)$ & & $39.5(32.5-47)$ & \\
\hline Good & $36(29-48)$ & & $33(28-45)$ & \\
\hline Relations with other patients: & & 0.175 & & 0.688 \\
\hline Very good & $39(34-56)$ & & $40(32-49)$ & \\
\hline Good & $35.5(28-48)$ & & $35(30.5-45)$ & \\
\hline Moderate & $32(23-48)$ & & $41(29-50)$ & \\
\hline Dependence on healthcare professionals: & & 0.123 & & 0.122 \\
\hline Very & $40(31-53)$ & & $40.5(32-51)$ & \\
\hline Enough & $36(27-44)$ & & $35.5(29-45)$ & \\
\hline Support from family: & & 0.205 & & 0.203 \\
\hline Very much & $36(27-48)$ & & $40(30-49)$ & \\
\hline Enough & $39.5(31.5-53)$ & & $34(31.5-39)$ & \\
\hline Satisfied with life: & & 0.001 & & 0.001 \\
\hline Very much & $26.5(23-36)$ & & $28.5(26-35)$ & \\
\hline Enough & $39(31-51)$ & & $38(32-45)$ & \\
\hline Little/not at all & $51(34-64)$ & & $49(44-51)$ & \\
\hline Insecure because you live on an island: & & 0.015 & & 0.238 \\
\hline Yes & $40.5(31-52)$ & & $39(30-51)$ & \\
\hline No & $32.5(24.5-38.5)$ & & $36.5(31.5-43)$ & \\
\hline
\end{tabular}

spectively). In addition, patients who had limited very much or enough their social contact (median 47 and 43, respectively), as well as patients who were little or not at all satisfied with their lives (median 51 and 49, respectively), had more state and trait anxiety.

In addition, patients' state anxiety was found to be statistically significantly associated with the insecurity they felt due to their living on an island $(p=0.015)$, whereas trait anxiety was found to be statistically significantly associated with educational level ( $p=0.003)$. Patients feeling insecurity because they were living on an island had more state anxiety (median 40.5) than patients without such insecurity (median 32.5). Similarly, patients with primary education (median 43) had more trait anxiety than those with secondary and higher education (median 36 and 32.5, respectively).

\section{Association between patients'} characteristics and perceived social support

Table VII presents the association between patients' characteristics and social support.

A statistically significant association was observed between the score of support from significant ones and marital status $(p=0.001)$, insomnia $(p=0.026)$, limitation of social contact ( $p=$ $0.011)$, and satisfaction from life $(p=0.001)$. In greater detail, married patients experienced statistically significantly higher levels of support from significant ones (median 28) than single patients (median 24). Those who did not have insomnia had statistically significantly higher levels of support from significant ones (median 28) compared to those with insomnia (median 25). Patients who had not limited or had only slightly limited their 
Table VII. Association between patients' characteristics and perceived social support

\begin{tabular}{|c|c|c|c|c|c|c|}
\hline Parameter & $\begin{array}{c}\text { Significant } \\
\text { ones } \\
\text { Median (IQR) }\end{array}$ & $P$-value & $\begin{array}{c}\text { Family } \\
\text { Median (IQR) }\end{array}$ & $P$-value & $\begin{array}{c}\text { Friends } \\
\text { Median (IQR) }\end{array}$ & $P$-value \\
\hline Gender: & & 0.314 & & 0.893 & & 0.548 \\
\hline Male & $28(24-28)$ & & $27(24-28)$ & & $20(18-26)$ & \\
\hline Female & $26(23-28)$ & & $27(23-28)$ & & $20(16-25)$ & \\
\hline Age: & & 0.729 & & 0.463 & & 0.013 \\
\hline$<50$ & $27(24-28)$ & & $28(25-28)$ & & $25(21-28)$ & \\
\hline $51-60$ & $28(25-28)$ & & $28(27-28)$ & & $19(15-24)$ & \\
\hline $61-70$ & $27.5(22.5-28)$ & & $27(24-28)$ & & $21(19-25)$ & \\
\hline$>70$ & $26(23-28)$ & & $27(23-28)$ & & $17(15-24)$ & \\
\hline Family status: & & 0.001 & & 0.021 & & 0.536 \\
\hline Married/living together & $28(25-28)$ & & $28(25-28)$ & & $21(16-27)$ & \\
\hline Single/widowed/divorced & $24(21-26)$ & & $25(23-28)$ & & $20(16-24)$ & \\
\hline Education: & & 0.329 & & 0.181 & & 0.001 \\
\hline Primary school & $25(23-28)$ & & $26(23-28)$ & & $18(15-22)$ & \\
\hline High school & $27(24-28)$ & & $28(25-28)$ & & $20(16-26)$ & \\
\hline University & $28(25-28)$ & & $28(24-28)$ & & $24.5(24-28)$ & \\
\hline Job: & & 0.073 & & 0.058 & & 0.001 \\
\hline Unemployed/household & $23.5(21-27)$ & & $24.5(21.5-27)$ & & $15.5(14.5-19)$ & \\
\hline Employee & $28(25-28)$ & & $28(25-28)$ & & $24(20-28)$ & \\
\hline Pensioner & $26(23-28)$ & & $27(24-28)$ & & $20(16-26)$ & \\
\hline Cardiac disease: & & 0.609 & & 0.309 & & 0.862 \\
\hline Heart failure & $26(24-28)$ & & $27(26-28)$ & & $22(17-28)$ & \\
\hline Myocardial infraction & $28(23.5-28)$ & & $28(24-28)$ & & $20(16.5-26.5)$ & \\
\hline Atrial fibrillation & $28(24.5-28)$ & & $27.5(25-28)$ & & $20.5(16.5-25.5)$ & \\
\hline Unstable angina & $26(21-28)$ & & $25(20.5-27.5)$ & & $19(16-24.5)$ & \\
\hline Pacemaker & $23(20-28)$ & & $23(21-28)$ & & $20(15-28)$ & \\
\hline $\begin{array}{l}\text { Number of years since the } \\
\text { onset of cardiac disease: }\end{array}$ & & 0.112 & & 0.155 & & 0.055 \\
\hline$<1$ & $28(26-28)$ & & $28(27-28)$ & & $24.5(20-28)$ & \\
\hline $2-5$ & $27(22-28)$ & & $27(24-28)$ & & $21(18-25)$ & \\
\hline $6-15$ & $25(22.5-28)$ & & $26(23-28)$ & & $16(15-20.5)$ & \\
\hline$>15$ & $26(24-28)$ & & $27(23-28)$ & & $19(17-24)$ & \\
\hline Other disease: & & 0.131 & & 0.034 & & 0.003 \\
\hline Yes & $26(23-28)$ & & $27(23.5-28)$ & & $20(16-24.5)$ & \\
\hline No & $28(27-28)$ & & $28(28-28)$ & & $28(25-28)$ & \\
\hline Insomnia: & & 0.026 & & 0.032 & & 0.033 \\
\hline Yes & $25(22-28)$ & & $25.5(23-28)$ & & $18(15.5-25)$ & \\
\hline No & $28(24-28)$ & & $28(25-28)$ & & $22.5(18-27)$ & \\
\hline $\begin{array}{l}\text { Level of information } \\
\text { about cardiac disease: }\end{array}$ & & 0.755 & & 0.569 & & 0.464 \\
\hline High & $26(23-28)$ & & $28(25-28)$ & & $23(16-28)$ & \\
\hline Adequate & $28(24-28)$ & & $27(23-28)$ & & $20.5(16-26)$ & \\
\hline Low & $26(23-28)$ & & $27(21-28)$ & & $18(17-20)$ & \\
\hline $\begin{array}{l}\text { Need of receiving written } \\
\text { information: }\end{array}$ & & 0.314 & & 0.445 & & 0.084 \\
\hline High & $26.5(23-28)$ & & $27(24-28)$ & & $20(16-25.5)$ & \\
\hline Moderate & $28(25-28)$ & & $28(26-28)$ & & $24(19-28)$ & \\
\hline Low & $24.5(23-28)$ & & $27.5(21.5-28)$ & & $17(15-22.5)$ & \\
\hline
\end{tabular}


Table VII. Cont.

\begin{tabular}{|c|c|c|c|c|c|c|}
\hline Parameter & $\begin{array}{l}\text { Significant } \\
\text { ones } \\
\text { Median (IQR) }\end{array}$ & $P$-value & $\begin{array}{c}\text { Family } \\
\text { Median (IQR) }\end{array}$ & $P$-value & $\begin{array}{c}\text { Friends } \\
\text { Median (IQR) }\end{array}$ & $P$-value \\
\hline $\begin{array}{l}\text { Difficulties in everyday } \\
\text { activities: }\end{array}$ & & 0.086 & & 0.137 & & 0.238 \\
\hline Yes & $25(22-28)$ & & $26(23-28)$ & & $20(16-26)$ & \\
\hline No & $28(24-28)$ & & $28(25-28)$ & & $21(18-26)$ & \\
\hline Limited social contacts: & & 0.011 & & 0.104 & & 0.019 \\
\hline Very/enough & $23.5(21.5-26)$ & & $25(22.5-27.5)$ & & $16(15-20.5)$ & \\
\hline Little & $28(24.5-28)$ & & $28(26.5-28)$ & & $21.5(16.5-28)$ & \\
\hline Not at all & $27(24-28)$ & & $27(24-28)$ & & $22(19-25)$ & \\
\hline $\begin{array}{l}\text { Relations with nursing } \\
\text { staff: }\end{array}$ & & 0.903 & & 0.898 & & 0.709 \\
\hline Very good & $27(24-28)$ & & $27(24-28)$ & & $20(16-26)$ & \\
\hline Good & $28(22-28)$ & & $27.5(22.5-28)$ & & $20(16.5-28)$ & \\
\hline $\begin{array}{l}\text { Relations with medical } \\
\text { staff: }\end{array}$ & & 0.355 & & 0.421 & & 0.234 \\
\hline Very good & $26(23.5-28)$ & & $27(24-28)$ & & $20(16-26)$ & \\
\hline Good & $28(22-28)$ & & $28(24-28)$ & & $24(18-28)$ & \\
\hline $\begin{array}{l}\text { Relations with other } \\
\text { patients: }\end{array}$ & & 0.339 & & 0.197 & & 0.241 \\
\hline Very good & $26(24-28)$ & & $28(25-28)$ & & $22(16-28)$ & \\
\hline Good & $28(23.5-28)$ & & $27.5(23.5-28)$ & & $21.5(17-26.5)$ & \\
\hline Moderate & $24(22-28)$ & & $25(21-28)$ & & $19(15-21)$ & \\
\hline $\begin{array}{l}\text { Dependence on } \\
\text { healthcare professionals: }\end{array}$ & & 0.888 & & 0.697 & & 0.268 \\
\hline Very & $27(23-28)$ & & $27(23-28)$ & & $20(16-26)$ & \\
\hline Enough & $27(24-28)$ & & $27.5(25-28)$ & & $20.5(18-28)$ & \\
\hline Satisfied with life: & & 0.001 & & 0.001 & & 0.002 \\
\hline Very & $28(28-28)$ & & $28(28-28)$ & & $23.5(19-28)$ & \\
\hline Enough & $27(23-28)$ & & $27(24-28)$ & & $21(17-27)$ & \\
\hline Little/not at all & $23(20-25)$ & & $23(21-27)$ & & $16(15-20)$ & \\
\hline $\begin{array}{l}\text { Insecure because you live } \\
\text { on an island: }\end{array}$ & & 0.416 & & 0.836 & & 0.484 \\
\hline Yes & $26(23-28)$ & & $27(24-28)$ & & $20(16-26)$ & \\
\hline No & $28(24-28)$ & & $27(24-28)$ & & $20(17.5-27.5)$ & \\
\hline
\end{tabular}

social contact due to cardiac problems had higher levels of support from significant ones (median 27 and 28, respectively) than those who had limited them a lot (median 23.5). In addition, patients who were very satisfied with their lives experienced higher levels of support from significant ones (median 28).

A statistically significant association was observed between the score of support from family and family status $(p=0.021)$, other diseases $(p=$ $0.034)$, insomnia ( $p=0.032)$, and satisfaction with life $(p=0.001)$. More specifically, married patients experienced statistically significantly higher levels of support from family (median 28) than single patients (median 25). Those without another dis- ease had statistically significantly higher levels of support from family (median 28) than those with another disease (median 27). Those without insomnia also had statistically significantly higher levels of support from family (median 28) than those with insomnia (median 25.5). Patients who were very or adequately satisfied with their life had higher levels of support from family (median 28 and 27, respectively) than those who were less satisfied (median 23).

A statistically significant association was observed between scores of support from friends and age $(p=0.013)$, educational level $(p=0.001)$, occupation $(p=0.001)$, other diseases $(p=0.003)$, insomnia ( $p=0.019)$, and satisfaction from life 
$(p=0.002)$. More specifically, patients younger than 50 years experienced statistically significantly higher levels of support from friends (median 25) than patients older than 70 years (median 17). Patients with university education experienced statistically significantly higher levels of support from friends (median 24.5) than patients with primary level education (median 18). Employees also experienced statistically significantly higher levels of support from friends (median 24) than retired and unemployed patients (median 20 and 15.5, respectively). Those without another disease had statistically significantly higher levels of support from friends (median 28) than those with another disease (median 20). Furthermore, patients without insomnia had statistically significantly higher levels of support from friends (median 22.5) than those with insomnia (median 18). Lastly, patients who were very or adequately satisfied with their life had higher levels of support from friends (median 23.5 and 21 , respectively) than those who were less satisfied (median 16).

\section{Association between patients' anxiety and perceived social support}

Table VIII presents the association between patients' state and trait anxiety and the social support they receive from significant ones, family, and friends. We can observe that all associations were statistically significant $(p<0.05)$. The association between anxiety and social support was found to be negative, meaning that an increase in the score of social support either by significant ones, family, or friends leads to a decrease in the score of state and trait anxiety. In other words, the more support a patient felt, the less anxiety they experienced.

\section{The impact of patients' characteristics and social support on anxiety}

Multiple linear regression was performed to assess the effect of characteristics and of social support on patients' state and trait anxiety. The results are presented in Table IX. Patients who stated that they were adequately satisfied with their life and those who stated little or no sat- isfaction had higher state anxiety by 10.21 and 14.42 points, respectively, than patients who stated that they were very satisfied $(\beta=10.21$, 95\% Cl: 3.54-16.88, $p=0.003$ and $\beta=14.42$, $95 \% \mathrm{Cl}: 4.55-24.29, p=0.005)$. Similarly, patients who were adequately or a little satisfied with their life had 5.56 and 10.26 points higher trait anxiety, respectively, than those who were very satis fied $(\beta=5.56,95 \% \mathrm{Cl}: 0.60-10.51, p=0.029$ and $\beta=10.26$; $95 \% \mathrm{Cl}: 2.97-17.55, p=0.006)$. In addition, patients who had slightly limited their social contact had 7.7 points higher trait anxiety than patients who had limited their social contact a lot $(\beta=7.70,95 \% \mathrm{Cl}: 1.24-14.16, p=0.020)$.

\section{Discussion}

The results of the present study show moderate levels of both trait and state anxiety among islanders with cardiac disease.

Anxiety seems to be present in several forms of cardiac disease. High levels of anxiety were observed in $34.9 \%$ of outpatients with atrial fibrillation [15], $24.7 \%$ of patients with heart failure [16], $27.2 \%$ [17] of pacemaker patients, and $20-40 \%$ [18] of cardioverter defibrillator patients.

All these figures have brought early screening for anxiety to the forefront of clinical care [1].

The main sources of anxiety in cardiac patients include complex therapeutic regimen, comorbidities, failure of coping mechanisms, frustrations with complicated healthcare system, financial worries, fear of death, hopelessness, and social isolation [1]. On the other hand, anxiety is associated with poor clinical outcomes, such as failure to participate in rehabilitation programs, recurrent cardiac events, poor quality of life, and unhealthy lifestyle behaviour (physical inactivity, smoking, and inappropriate diet) [1, 2]. Alleviating anxiety is crucial because the more anxiety cardiac patients feel, the worse their quality of life [19].

Furthermore, regarding clinical characteristics, both state and trait anxiety was associated with the existence of other disease and insomnia. Interestingly, anxiety and insomnia seem to be part of a vicious circle in which sleep deprivation may increase the risk of anxiety while insomnia may deteriorate anxiety or prevent recovery [20, 21]. Apart from this association, insomnia raises sig-

Table VIII. Association of anxiety and social support

\begin{tabular}{|lcccc|}
\hline Support from & \multicolumn{2}{c}{ State anxiety } & \multicolumn{2}{c|}{ Trait anxiety } \\
\cline { 2 - 5 } & Spearman's Rho & $P$-value & Spearman's Rho & $P$-value \\
\hline Significant ones & -0.235 & 0.033 & -0.488 & 0.001 \\
\hline Family & -0.271 & 0.014 & -0.438 & 0.001 \\
\hline Friends & -0.266 & 0.016 & -0.434 & 0.001 \\
\hline
\end{tabular}


Table IX. Effect of characteristics and social support on patients' anxiety

\begin{tabular}{|c|c|c|c|c|}
\hline Parameter & $\begin{array}{c}\text { State anxiety } \\
\beta(95 \% \mathrm{Cl})\end{array}$ & $P$-value & $\begin{array}{c}\text { Trait anxiety } \\
\beta(95 \% \mathrm{Cl})\end{array}$ & $P$-value \\
\hline \multicolumn{5}{|l|}{ Education: } \\
\hline Primary school & - & & Ref. cat. & \\
\hline High school & - & & $-2.00(-6.63-2.63)$ & 0.392 \\
\hline University & - & & $-5.68(-11.82-0.46)$ & 0.069 \\
\hline \multicolumn{5}{|l|}{ Other disease: } \\
\hline No & Ref. cat. & & Ref. cat. & \\
\hline Yes & $2.99(-6.11-12.09)$ & 0.514 & $3.28(-3.06-9.62)$ & 0.306 \\
\hline \multicolumn{5}{|l|}{ Insomnia: } \\
\hline No & Ref. cat. & & Ref. cat. & \\
\hline Yes & $0.36(-5.97-6.69)$ & 0.911 & $-1.51(-6.22-3.21)$ & 0.526 \\
\hline \multicolumn{5}{|c|}{ Difficulties in everyday activities: } \\
\hline No & Ref. cat. & & Ref. cat. & \\
\hline Yes & $5.02(-1.03-11.08)$ & 0.103 & $3.51(-1.20-8.23)$ & 0.142 \\
\hline \multicolumn{5}{|c|}{ Limited social contact: } \\
\hline Very/enough & Ref. cat. & & Ref. cat. & \\
\hline Little & $5.61(-3.10-14.31)$ & 0.203 & $7.70(1.24-14.16)$ & 0.020 \\
\hline Not at all & $-2.30(-10.73-6.13)$ & 0.588 & $0.37(-5.75-6.49)$ & 0.905 \\
\hline \multicolumn{5}{|l|}{ Satisfied with life: } \\
\hline Very & Ref. cat. & & Ref. cat. & \\
\hline Enough & $10.21(3.54-16.88)$ & 0.003 & $5.56(0.60-10.51)$ & 0.029 \\
\hline Little/not at all & $14.42(4.55-24.29)$ & 0.005 & $10.26(2.97-17.55)$ & 0.006 \\
\hline \multicolumn{5}{|c|}{ Insecure because you live on an island: } \\
\hline No & Ref. cat. & & - & \\
\hline Yes & $5.59(-0.39-11.56)$ & 0.066 & - & \\
\hline \multicolumn{5}{|l|}{ Support from: } \\
\hline Significant ones & $-0.30(-1.90-1.30)$ & 0.712 & $-1.14(-2.33-0.05)$ & 0.061 \\
\hline Family & $-0.05(-1.14-1.03)$ & 0.920 & $0.05(-0.77-0.86)$ & 0.910 \\
\hline Friends & $-0.16(-0.71-0.39)$ & 0.561 & $-0.07(-0.51-0.38)$ & 0.767 \\
\hline
\end{tabular}

nificant concerns within the field of cardiac diseases. It was nearly two decades ago when it was shown that sleep complaints increase the likelihood of a first myocardial infarction in adults $>65$ years old, independently of classic coronary risk factors [22]. More recently, Mallon et al. [23], in a 12-year follow-up, showed that subjective sleep complaints may predict coronary artery disease mortality in middle-aged males.

Cardiac patients suffering from insomnia should undergo specific evaluation and treatment because improvement in insomnia is associated with improvements not only in anxiety but also in total cholesterol and depression after completion of a 12-week cardiac rehabilitation programme [24]. Another commonly held view is that insomnia among cardiac patients is attributed to ongoing stressors or may be part of a syndrome involving depression [20, 21].
Results also revealed that difficulties in daily activities and limitations in social contact were associated with both state and trait anxiety. Cardiovascular disease is associated with limitations to physical functioning, inability to continue in prior rhythms, and loss of independent living, thus triggering increased levels of anxiety [25].

On the other hand, psychological variables such as anxiety may affect cardiac patients' performance [26]. For instance, a one point increase in anxiety implied a significant deterioration in the physical and mental state by 0.57 and 0.39 points, respectively, among 100 heart failure outpatients (64 men and 36 women) [27]. Also, anxiety independently predicts a decline in physical function over 6 months in heart failure patients [28]. Restrictions in functional level represent a precursor to disability and lowering life expectancy [29]. Conversely, anxiety may prevent patients from re- 
turning to activities that they perceive as a source of stress, even though they may be beneficial [30].

Moreover, in terms of patients' beliefs, those who reported dissatisfaction with their life experienced greater anxiety. One proposed explanation is that patients perceive no control over their illness. Personal control among heart transplantation patients was positively correlated with satisfaction with life and negatively correlated with anger and depression [31]. Patients' perceptions are a significant area of interest, which may influence all stages of a cardiac disease. Indeed, patients' views and options may play a vital role in changing personal behaviours, following recommended treatment, and rehabilitation. Exploring patients' perspectives about illness may shed more light on disease management [30].

In addition, patients reporting insecurity because they lived on island had more transient anxiety. Individuals living in the province lack immediate access to specialised medical help or may encounter transportation issues, especially in winter. Given that care of cardiac disease requires frequent contact with the healthcare system, telemedicine might be an alternative form of treatment. Interestingly, this method may minimise hospitalisation or access to emergency services, reduce the burden of traveling to and from treatment centres, and thus ultimately reduce anxiety [32].

Analysis of data showed high levels of support from family, friends, or significant others. Similarly, a high level of support among 1550 middle-aged people with implanted defibrillator was shown by Allemann et al. [33], whereas living alone was a major predictor of low/medium support.

Results also revealed that an increase in support implied a reduction in anxiety. The presence of supportive relatives among Danish cardiac patients having ischaemic disease, atrial fibrillation, heart failure, or valve disease was associated with lower frequency of anxiety [34]. Moreover, social support was linked to their psychosocial adjustment to coronary heart disease [35].

To the best of our knowledge, there are no related data from other areas that might permit comparisons between islander populations. Given that the interaction of environmental, behavioural, and clinical characteristics may determine the span of life, as in the island of Ikaria [36], then exploring social support among cardiac islanders might be a new area of interest.

Several limitations of our study must be acknowledged. First, convenience sampling is one of the limitations because this method is not representative of all the islanders with cardiac disease in Greece, thus limiting the generalisability of the results. The sample size was relatively small, although many significant associations were observed. Furthermore, there was no longitudinal design with follow-up data on the same patients, which may permit evaluation of possible changes in all dimensions (anxiety and perceived social support). It would be interesting to monitor anxiety 12 or 24 months after baseline. Self-report questionnaires are not considered sufficiently precise to make a psychiatric diagnosis of anxiety.

The strengths of the study include the use of the widespread STAI instrument, which may permit comparisons among cardiac populations across the world.

In conclusion, more both state and trait anxiety experienced patients who reported to have some other disease, to have insomnia, to limit social contacts and those who were not satisfied by their lives.

An increase of social support either by significant ones, family, or friends leads to a decrease in state and trait anxiety. In other words, the more support a patient felt, the less anxiety they experienced.

Stakeholders may use the evidence provided in this research and support tangible action to improve cardiac health in island areas.

\section{Conflict of interest}

The authors declare no conflict of interest.

\section{References}

1. Polikandrioti $M$, Olympios C. Anxiety and coronary artery disease. Arch Hell Med 2014; 31: 403-11.

2. Celano CM, Daunis DJ, Lokko HN, et al. Anxiety disorders and cardiovascular disease. Curr Psychiatry Rep 2016; 18: 101.

3. Palacios J, Khondoker M, Mann A, et al. Depression and anxiety symptom trajectories in coronary heart disease: associations with measures of disability and impact on 3-year health care costs. J Psychosom Res 2018; 104: $1-8$.

4. Alexandri A, Georgiadi E, Mattheou P, et al. Factors associated with anxiety and depression in hospitalized patients with first episode of acute myocardial infarction. Arch Med Sci Atheroscler Dis 2017; 2: e90-9.

5. Grace SL, Abbey SE, Irvine J, et al. Prospective examination of anxiety persistence and its relationship to cardiac symptoms and recurrent cardiac events. Psychother Psychosom 2004; 73: 344-52.

6. Cohen BE, Edmondson D, Kronish IM. State of the art review: depression, stress, anxiety, and cardiovascular disease. Am J Hypertens 2015; 28: 1295-302.

7. Julian LJ. Measures of anxiety: State-Trait Anxiety Inventory (STAI), Beck Anxiety Inventory (BAI), and Hospital Anxiety and Depression Scale-Anxiety (HADS-A). Arthritis Care Res 2011; 63 Suppl 11: S467-72.

8. Lilympaki I, Makri A, Vlantousi K, et al. Effect of perceived social support on the levels of anxiety and depression of hemodialysis patients. Mater Sociomed 2016; 28: 361-5.

9. Laopoulou F, Kelesi M, Fasoi G, et al. Perceived social support in individuals with diabetic foot ulcers: a cross-sectional survey. J Wound Ostomy Continence Nurs 2020; 47: 65-71. 
10. Blikman MJ, Jacobsen HR, Eide GE, et al. How important are social support, expectations and coping patterns during cardiac rehabilitation. Rehabil Res Pract 2014; 2014: 973549.

11. Compare A, Zarbo C, Manzoni GM, et al. Social support, depression, and heart disease: a ten year literature review. Front Psychol 2013; 4: 384.

12. Foscolou A, Polychronopoulos E, Paka E, et al. Lifestyle and health determinants of cardiovascular disease among Greek older adults living in Eastern Aegean Islands: an adventure within the MEDIS study. Hellenic J Cardiol 2016; 57: 407-14.

13. Fountoulakis KN, Papadopoulou M, Kleanthous S, et al. Reliability and psychometric properties of the Greek translation of the State-Trait Anxiety Inventory form Y: preliminary data. Ann Gen Psychiatry 2006; $5: 2$.

14. Theofilou P. Translation and cultural adaptation of the multidimensional scale of perceived social support for Greece. Health Psychol Res 2015; 3: 1061.

15. Polikandrioti M, Koutelekos I, Vasilopoulos G, et al. Anx iety and depression in patients with permanent atria fibrillation: prevalence and associated factors. Cardio Res Pract 2018; 2018: 7408129.

16. Polikandrioti M, Goudevenos J, Michalis LK, et al. Factors associated with depression and anxiety of hospitalized patients with heart failure. Hellenic J Cardiol 2015; 56 26-35.

17. Polikandrioti M, Tzirogiannis K, Zyga S, et al. Effect of anxiety and depression on the fatigue of patients with a permanent pacemaker. Arch Med Sci Atheroscler Dis 2018; 3: e8-17.

18. Magyar-Russell G, Thombs BD, Cai JX, et al. The prevalence of anxiety and depression in adults with implantable cardioverter defibrillators: a systematic review. J Psychosom Res 2011; 71: 223-31.

19. Polikandrioti M, Koutelekos I, Panoutsopoulos G, et al. Hospitalized patients with heart failure: the impact of anxiety, fatigue, and therapy adherence on quality of life. Arch Med Sci Atheroscler Dis 2019; 4: 268-79.

20. Spiegelhalder K, Scholtes C, Riemann D. The association between insomnia and cardiovascular diseases. Nat Sci Sleep 2010; 2: 71-8.

21. Malhotra A, Loscalzo J. Sleep and cardiovascular disease: an overview. Prog Cardiovasc Dis 2009; 51: 279-84.

22. Schwartz SW, Cornoni-Huntley J, Cole SR, et al. Are sleep complaints an independent risk factor for myocardial infarction? Ann Epidemiol 1998; 8: 384-92.

23. Mallon L, Broman JE, Hetta J. Sleep complaints predict coronary artery disease mortality in males: a 12-year follow-up study of a middle-aged Swedish population. J Intern Med 2002; 251: 207-16.

24. Rouleau CR, Toivonen K, Aggarwal S, et al. The association between insomnia symptoms and cardiovascular risk factors in patients who complete outpatient cardiac rehabilitation. Sleep Med 2017; 32: 201-7.

25. Britton A, Brunnner E, Kivimaki M, et al. Limitations to functioning and independent living after the onset of coronary heart disease: what is the role of lifestyle factors and obesity? Eur J Public Health 2012; 22: 831-5.

26. Allahverdipour $\mathrm{H}$, Asgharijafarabadi $M$, Heshmati $\mathrm{R}$, et al. Functional status, anxiety, cardiac self-efficacy, and health beliefs of patients with coronary heart disease. Health Promot Perspect 2013; 3: 217-29.

27. Polikandrioti M, Panoutsopoulos G, Tsami A, et al. Assessment of quality of life and anxiety in heart failure outpatients. Arch Med Sci Atheroscler Dis 2019; 4: e38-46.
28. Shen BJ, Eisenberg SA, Maeda U, et al. Depression and anxiety predict decline in physical health functioning in patients with heart failure. Ann Behav Med 2011; 41: 373-82.

29. Keeler E, Guralnik JM, Tian H, et al. The impact of functional status on life expectancy in older persons. J Gerontol A Biol Sci Med Sci 2010; 65: 723-33.

30. Tinetti ME, Naik AD, Dodson JA. Moving from disease-centered to patient goals-directed care for patients with multiple chronic conditions: patient valuebased care. JAMA Cardiol 2016; 1: 9-10.

31. Bohachick P, Taylor MV, Sereika S, et al. Social support, personal control, and psychosocial recovery following heart transplantation. Clin Nurse Res 2002; 11: 34-51.

32. Di Lenarda A, Casolo G, Gulizia MM, et al. The future of telemedicine for the management of heart failure patients: a Consensus Document of the Italian Association of Hospital Cardiologists (A.N.M.C.O), the Italian Society of Cardiology (S.I.C.) and the Italian Society for Telemedicine and eHealth (Digital S.I.T.). Eur Heart J 2017; Suppl D: D113-29.

33. Allemann H, Strömberg A, Thylén I. Perceived social support in persons with heart failure living with an implantable cardioverter defibrillator: a cross-sectional explorative study. J Cardiovasc Nurs 2018; 33: E1-8.

34. Hansen C, Zinckernagel L, Schneekloth N, et al. The association between supportive relatives and lower occurrence of anxiety and depression in heart patients: results from a nationwide survey. Eur J Cardiovasc Nurs 2017; 16: 733-41.

35. Karataş T, Bostanoğlu H. Perceived social support and psychosocial adjustment in patients with coronary heart disease. Int J Nurs Pract 2017; 23. doi: 10.1111/ ijn.12558.

36. Panagiotakos DB, Chrysohoou C, Siasos G, et al. Sociodemographic and lifestyle statistics of oldest old people (> 80 years) living in Ikaria island: the ikaria study. Cardiol Res Pract 2011; 2011: 679187. 